\title{
RESEARCH
}

Open Access

\section{The role of host soluble inflammatory mediators induced by the BCG vaccine for the initiation of in vitro monocyte apoptosis in healthy Brazilian volunteers}

\author{
Jessica Lima ${ }^{\dagger}$, Mariana Siqueira ${ }^{\dagger}$, Thaíze Pedro, Carlos Ponte, Leandro Peres, Suelen Marinho,
} Luíz R. Castello-Branco and Paulo R. Z. Antas ${ }^{*+}$

\begin{abstract}
Background: Tuberculosis (TB) is the second greatest killer worldwide that is caused by a single infectious agent. For its control, studies of TB vaccines are needed. Since Bacillus Calmette-Guerin (BCG) is the only vaccine against TB currently in use, studies addressing the protective role of BCG in the context of inducible inflammatory mediators are urgently required.

Methods: In this study, groups of HIV-negative adult healthy donors (HD; $n=42)$ and neonates $(U V ; n=18)$ have been voluntarily enrolled, and BCG Moreau strain was used for the in vitro mononuclear cell infections for an initial period of $48 \mathrm{~h}$. Subsequently, harvested conditioned medium (CM) was added to autologous resting cells for an additional 24, 48, and $120 \mathrm{~h}$, and Annexin V, in conjunction with a vital dye, was then used for apoptosis detection. $\mathrm{CM}$ was also assayed for nitric oxide (NO), prostaglandin E2 (PGE $)$, leukotriene B4 (LTB 4 ), interferon (IFN)- $\beta$, and transforming growth factor (TGF)- $\beta 1$ levels. The $p$ values were set up for any differences between two groups of individuals using Student's $t$-test and considered significant when $\leq 0.05$.

Results: At 120 h, CM induced the highest apoptosis levels in both group studied, but necrosis was high in UV group only ( $p$-value $<0.05$ ). NO was released equally during BCG infection in both groups, but higher levels were found in HD when compared with UV group ( $p$-value < 0.05). Overall, BCG Moreau triggered high $\mathrm{PGE}_{2}, \mathrm{LTB}_{4}$ and IFN- $\beta$ productions in macrophages from the UV group ( $p$-value $\leq 0.05$ ), whereas the prostanoid PGE 2 and TGF- $\beta 1$ had an opposite pattern in the HD group.

Conclusions: This study uncovers critical roles for endogenous compounds in the instruction of host macrophage cell death patterns. Understanding the regulation of human immune responses is critical for vaccine development and the treatment of infectious diseases. These findings shed new light on the potential condition for a booster immunization in individuals already vaccinated with BCG for TB protection, and further studies are warranted.
\end{abstract}

Keywords: BCG vaccine, Tuberculosis, Monocyte, Apoptosis, Cytokine, Inflammation, Conditioned medium

\footnotetext{
* Correspondence: pzuquim@ioc.fiocruz.br

${ }^{\dagger}$ Equal contributors

Laboratório de Imunologia Clínica, Instituto Oswaldo Cruz, Fiocruz, Rio de Janeiro, Brazil
} 


\section{Background}

Despite the continuous evolution of better management, new drug development, and additional diagnostic tools available in public health, tuberculosis (TB) still remains as a serious global problem. There were a total of 8.6 million new cases and 1.3 million deaths in 2012; TB is a leading killer of people living with HIV [1]. It is currently estimated that one third of the world population is infected with Mycobacterium tuberculosis and that $5-10 \%$ of those infected will develop the disease during their life-time [2].

The current TB vaccine is $M$. bovis bacille CalmetteGuérin (BCG), and it has been employed for nearly a century to prevent the disease. However, while BCG is the most common and broadly used vaccine worldwide, its efficacy remains quite controversial: In endemic sites, the vaccine has evoked unpredictable outcomes [3]. Epidemiological studies point to BCG as affording more complete protection against the meningeal and miliary clinical forms of TB in children than against the pulmonary clinical form in adults (Reviewed by [4]). It is generally perceived that the BCG vaccine is not fully effective because of the absence of antigens commonly shared with $M$. tuberculosis, but also that the rapid removal of BGC via systemic immunity, primarily targeting atypical environmental mycobacteria, reduces the vaccine's effectiveness over time. Therefore, more studies are required to better understand how BCG confers protection in humans.

Conventional studies have focused solely on the role of cytokines released by monocytes infected with BCG as a starting point for inducing immunity, such as the proinflammatory interleukin 1 beta (IL-1 $\beta$ ) and tumor necrosis factor alpha (TNF- $\alpha$ ) (Reviewed by $[5,6])$. Thus, one of the issues raised concerns the additional soluble compounds that are potentially secreted by host cells during BCG infection. Also, there may be a later increase in those factors for the initiation of apoptosis, necrosis, necroptosis and pyroptosis. The action of inflammatory caspases, such as caspase-1, has shown promising results for the activation of IL-1 $\beta$ through an inflammasome mechanism during microbial pathogens interactions [7].

Our prior study supports the hypothesis that BCG induces distinct cell-death patterns during the maturation of the immune system [8]. In the study, we observed increasing apoptosis in BCG-stimulated monocytes from healthy, vaccinated adults, associated with a release of IL-1 $\beta$ and TNF- $\alpha$, but not with metalloproteinase-9. Conversely, higher monocyte necrosis, but not apoptosis, was observed following the infection of umbilical vein cells from naïve neonates. This pattern was paralleled by different pro-inflammatory cytokine levels when compared to adults. In addition, necrosis has been defined as a mechanism used by virulent bacteria to exit macrophages, evade the host defenses, and disseminate; apoptosis has been associated with diminished pathogen viability $[9,10]$.

To address remaining critical issues beyond these premises, we have constructed a model of conditioned cell culture medium, i.e., culture supernatants obtained from host cells infected with BCG for $48 \mathrm{~h}$, for the study of the factors secreted by those cells that potentially are responsible for monocyte cell-death during the context of infection. Our hypothesis has a basis in compelling evidence from one recent study where eicosanoid lipid intermediaries regulated the cell death program of human macrophages infected with $M$. tuberculosis [10]. Thus, a cross-sectional population study of samples from Brazil was the means to uncover critical aspects of the in vitro degree of apoptosis and necrosis induced by BCG Moreau in monocytes from individuals, whether sensitized (adults) or non-sensitized (neonates). Using this method, there is input for a better understanding of the protective factors afforded by BCG against TB that would help to identify the processes by which this protection is achieved, thus opening up a horizon for its future improved clinical applicability.

\section{Methods}

\section{Study participants}

Between November 2010 and August 2012, two groups of donors were enrolled for this study at the Gaffrée Guinle State University Hospital of Rio de Janeiro (HUGG): healthy donor adults (HD; a total of 42 individuals) from the blood bank (anonymous donation policy, but included individuals age $\geq 18$-years old), and healthy mothers who participated in procedures to puncture umbilical cords and obtain blood samples from newborns' umbilical vein (UV; a total of 18 neonates). Inclusion and exclusion criteria for those HIV-seronegative individuals are described elsewhere [8]. The HUGG Institutional Review Board approved this study under protocols $\# 060 / 2009$ and $\# 089 / 2011$. All participants in this study provided written informed consent.

\section{Mononuclear cells purification, culture, in vitro infection with BCG and cell death assay}

The peripheral blood mononuclear cells (PBMC) and cord blood mononuclear cells (CBMC) were separated, cultured, and infected with $M$. bovis BCG Moreau strain for $48 \mathrm{~h}$ as previously described [8]. Cell cultures $\left(1 \times 10^{6}\right)$ were used at a multiplicity of infection (MOI) of 2:1 (bacilli:mononuclear cell). The bacilli viability was promptly assessed by a commercially available LIVE/DEAD BacLight bacterial viability kit (Life Techonologies, Carlsbad, CA, USA) in combination with flow cytometry. Next, the cell-free supernatants, henceforth called conditioned medium (CM), were collected, passed through a $0.22 \mu \mathrm{m}$ pore size filter (Millipore, Bedford, MA, USA), and instantly 
transferred $(700 \mu \mathrm{l})$ to either autologous resting PBMC or CBMC $\left(1 \times 10^{6}\right)$ for an additional 24, 48, and $120 \mathrm{~h}$. This followed a protocol similar to that of Nagabhushanam and colleagues [11]. Subsequent to incubation, cells were labeled using an apoptosis detection kit as specified by the manufacturer (TACS, R\&D systems, Minneapolis, $\mathrm{MN}, \mathrm{USA}$ ) and immediately analyzed in a flow cytometer device (FACScalibur, BD, San Jose, CA, USA). Those tubes designated as negative control (baseline) remained uninfected for the whole period of time. Other tubes designated as positive control consisted of cells subjected to a heating and boiling cycle just before staining in order to force cell necrosis [8]. A parallel process was used to establish the human acute monocytic leukemia cell line THP-1 (Kindly provided by Dr. M. C. V. Pessolani, FIOCRUZ, Brazil) in a monocyte-like state. Cells were grown, expanded, cultured in tubes $\left(1 \times 10^{6}\right)$, and then infected with $M$. bovis BCG Moreau strain (MOI of 2:1) for $48 \mathrm{~h}$. Next, cells were labeled and analyzed following the same apoptosis detection protocol as previously described.

\section{NO indirect detection by chemical test}

A modified Griess diazotization reaction for microassay detection of the presence of organic nitrite compounds, such as nitric oxide (NO), was used in thawed cell-free supernatants (or frozen CM) as previously described [12].

\section{$\mathrm{PGE}_{2}$, LTB ${ }_{4}$, IFN- $\beta$, TGF- $\beta 1$ detection by ELISA}

Prostaglandin E2 $\left(\mathrm{PGE}_{2}\right)$ and leukotriene $\mathrm{B} 4\left(\mathrm{LTB}_{4}\right)$, interferon (IFN) $-\beta$, and transforming growth factor (TGF) $-\beta 1$ levels were determined in thawed cell-free supernatants (or frozen $\mathrm{CM}$ ) using commercially available enzyme linked immunosorbent assay (ELISA) kits (Invitrogen Corp., Carlsbad, CA, USA; TFB Inc., Tokyo, Japan; and DuoSet, R\&D systems, Minneapolis, MN, USA, respectively). The immunoassays were carried out according to the manufacturer instructions. The detection limits were $30 \mathrm{pg} /$ $\mathrm{ml}\left(\mathrm{PGE}_{2}\right), 8 \mathrm{pg} / \mathrm{ml}\left(\mathrm{LTB}_{4}\right), 3.1 \mathrm{pg} / \mathrm{ml}(\mathrm{IFN}-\beta)$, and $20 \mathrm{pg} / \mathrm{ml}$ (TGF- $\beta 1$ ). For comparison purposes only, a thawed CM from BCG-infected mononuclears at $24 \mathrm{~h}$ was also included as an earlier time-point in both groups studied.

\section{Statistical evaluation}

Data were analyzed using Instat Statistical software, version 3.06 for Windows (GraphPad Software Inc., La Jolla, CA, USA). The significance of the difference in levels of immune responses between the two groups of individuals was calculated using the Student's $t$-test. The THP-1 cell-death levels were represented as the mean \pm standard error of the mean (SEM). The $p$-value was scored and considered significant when $\leq 0.05$.

\section{Results}

\section{Cell-Death ratio}

A better understanding of the pathways related to the in vitro cell-mediated immune responses in humans has been obtained for individuals, BCG-sensitized or not, which will assist in identifying the specific mechanisms that may confer protection against $M$. tuberculosis. To this end, and also based on our previous findings [8], we enrolled two groups of donors representing distinct immunity characteristics due to previous exposure to mycobacterial antigens. The first group consisted of healthy donor adults (HD) who had been already vaccinated with BCG Moreau during childhood. [Note: BCG vaccination in Brazil is universal after birth since 1967]. The second group consisted of naive individuals (i.e. neonates) who had never been exposed to mycobacteria; Umbilical vein (UV) cells from the naive individuals were promptly collected after their delivery. After standard experimental procedures [8], CM resembling cell-free supernatant was generated from 48 h BCG Moreauinfected cells (bacilli viability $>90 \%$ ), and the autologous remaining resting cultures were further incubated with $\mathrm{CM}$ for additional 24, 48 and $120 \mathrm{~h}$. Baseline cultures with no stimuli for a total of $168 \mathrm{~h}$ were used as a single negative control, since matched, earlier time-frames showed negligible differences. Cell-death events were analyzed to detect apoptosis and necrosis following an identical protocol described earlier [8]. Table 1 summarizes those findings.

We observed a significant increase in apoptosis ( $p$-value $<0.05)$ when $\mathrm{CM}$ was placed in contact with resting cells from both groups of individuals for an extended $120 \mathrm{~h}$, as compared to the baseline, negative control. Moreover, only cells from UV group showed an increase in necrosis $(p$-value $<0.05)$ under the same conditions and incubation periods. Curiously, in shorter experimental time-frames, there were no cell-death compared with negative control in both groups studied (Table 1). In addition, and in

Table 1 Specific cell-death (\%) in monocytes of healthy donor (HD, $n=16)$ and umbilical vein (UV, $n=5)$ groups after transference of conditioned medium (CM)

\begin{tabular}{llllll}
\hline & \multicolumn{3}{l}{ Apoptosis } & & \multicolumn{2}{l}{ Necrosis } \\
\cline { 2 - 3 } & HD & UV & & HD & UV \\
\hline Negative control & $32.5 \pm 4.2^{\mathrm{a}}$ & $12.7 \pm 2.1$ & & $12.2 \pm 2.5$ & $3.4 \pm 1.1$ \\
CM 24 h & $30.5 \pm 4.5$ & $14.8 \pm 1.2$ & $13.1 \pm 2.0$ & $2.4 \pm 0.4$ \\
CM 48 h & $36.1 \pm 4.1$ & $12.5 \pm 1.8$ & $12.7 \pm 2.1$ & $2.4 \pm 0.5$ \\
CM 120 h & $57.2 \pm 3.6^{\mathrm{b}}$ & $22.1 \pm 1.4^{\mathrm{b}}$ & & $12.4 \pm 1.7$ & $9.0 \pm 1.3^{\mathrm{b}}$ \\
Positive control $^{\mathrm{c}}$ & $31.8 \pm 5.4$ & $59.7 \pm 4.7^{\mathrm{b}}$ & & $57.4 \pm 5.3^{\mathrm{b}}$ & $36.2 \pm 4.8^{\mathrm{b}}$ \\
\hline
\end{tabular}

${ }^{\mathrm{a}}$ Mean \pm SEM;

$\mathrm{b}_{p}<0.05$, when compared to negative control (baseline):

${ }^{c}$ Heating samples was used to artificially induce necrosis 
opposition to the results described previously, the heating cycle caused an enhancement in apoptosis ( $p$-value $<0.05)$ only in the UV group, although necrosis $(p$-value $<0.05)$ was the highest in both groups, as expected.

With the aim of clarifying whether most of the primary monocytes increase in cell-death levels occurred after the $M$. bovis BCG infection, we used a smaller set of experiments $(n=6)$ to test the THP- 1 cell-line in our in vitro model. This step allowed us to confirm the previous results in primary mononuclear cell cultures $(17.1 \pm 1.0$ and $24.7 \pm 0.6 ; 6.5 \pm 0.6$ and $17.6 \pm 0.8$, at baseline and $48 \mathrm{~h}$ of BCG-infected THP-1 cell-line conditions for apoptosis and necrosis, respectively: $p$-values $<0.01)$. Thus, the THP-1 cell-line mirrored the cell-death patterns after BCG infection found particularly in the UV group studied.

Additionally, we attempted to better characterize the $\mathrm{CM}$ constituents in the next assays.

\section{Nitric Oxide levels}

The production of NO by host innate immune cells is recognized to be a successful defense against infection, but the specific mechanism by which $\mathrm{NO}$ antagonize the pathogen, involving induction of apoptosis in macrophages that harbor the mycobacteria, is not completely understood $[13,14]$. In addition, IL-1 $\beta$, TNF- $\alpha$, and mycobacterial cell wall components, along with T-cellderived IFN- $\gamma$, may also induce NO release. Thus, NO released during 48 h-BCG infection in both PBMC and CBMC cultures (the frozen $\mathrm{CM}$ ) were assayed, and the highest levels ( $p$-value $<0.01)$ were found only in the HD when compared with UV group (Fig. 1).

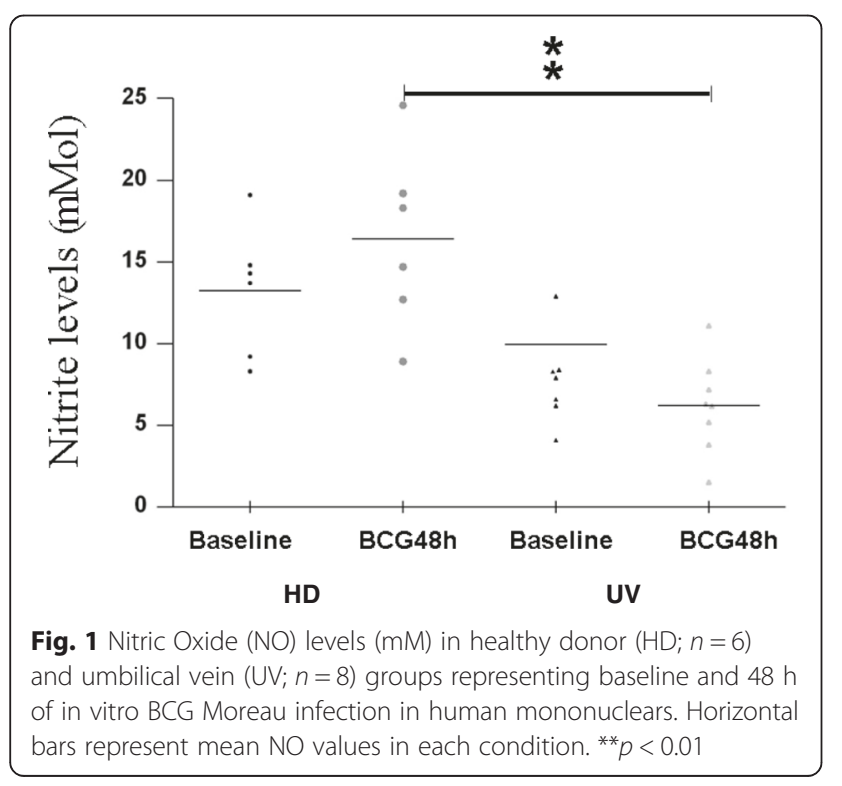

\section{Inflammatory lipid mediators and cytokines levels}

At an early encounter with noxious stimuli, the host produces a nonspecific inflammatory response, but a more specific immune response is believed to be modulated by two classes of molecules: eicosanoid lipid intermediaries, such as $\mathrm{PGE}_{2}$ and $\mathrm{LTB}_{4}$, and cytokines, synthesized by phagocytes [15]. A preceding study has associated increased prostanoid $\mathrm{PGE}_{2}$ levels with apoptosis, as well as high pronecrotic lipid mediators, such as $\mathrm{LTB}_{4}$ and necrosis [10]. Similarly, IFN- $\beta$ levels were shown to be elevated in necrosis [16] and necroptosis as well [17]. Importantly, increased $\mathrm{PGE}_{2}$ concentrations have been found in TB patients compared to HD and/or latent TB infected patients [18]. In order to uncover critical aspects of cell-death modality induced by BCG Moreau and to corroborate any potential link of those ecosanoids and IFN- $\beta$ with our in vitro cell-death system, competitive ELISA to the eicosanoids (Fig. 2), and ordinary ELISA to IFN- $\beta$ and TGF- $\beta 1$ were assayed (Fig. 3). When group means and dispersions for $\mathrm{PGE}_{2}$ in BCG-infected mononuclears were individually compared at 24 and $48 \mathrm{~h}$ to the baseline, a similar pattern was found in both groups studied (Fig. 2). However, the highest levels were achieved at $48 \mathrm{~h}$ only ( $p$-value $<$ $0.05)$. On the other hand, $\mathrm{LTB}_{4}$ was exclusively induced in UV group ( $p$-value $<0.05$; Fig. 2 ), whereas only a trend for higher levels after $24 \mathrm{~h}$ of the BCG-infected cells in the HD group was found ( $p$-value $=0.08$ ). In addition, the highest IFN $-\beta$ levels $(p$-value $\leq 0.05)$ did follow the ecosanoids pattern for the UV group only, but TGF- $\beta 1$ levels behaved as an opposite manner when compared to $\mathrm{PGE}_{2}$ in $\mathrm{HD}$ group, i.e. a drop in concentration after BCG-infected mononuclears ( $p$-value $\leq 0.05$; Fig. 3 ). Of note, when dispersions were compared between the 2 groups studied despite stimulus, UV group showed minimal range for TGF- $\beta 1$ levels. Besides, in order to highlight overall variations and particular changes to those soluble mediators at the individual donor level, connecting lines were built and thus allowed the visualization for a trend to mostly individuals tested (Additional file 1: Figure S1).

\section{Discussion}

Prophylactic medicine via vaccination is still the most cost effective means to reduce the spread of infectious diseases, and it also minimizes the burden on the health system. Because TB has imposed centuries-long threat to humans, the majority of the population in the tropics has been immunized with BCG in the infancy, but real protection against disease during adulthood has not yet been achieved. There is a long road ahead to better understand the entire clinical utility of BCG Moreau vaccine, and currently this task is our primary goal (Reviewed by [4]). 


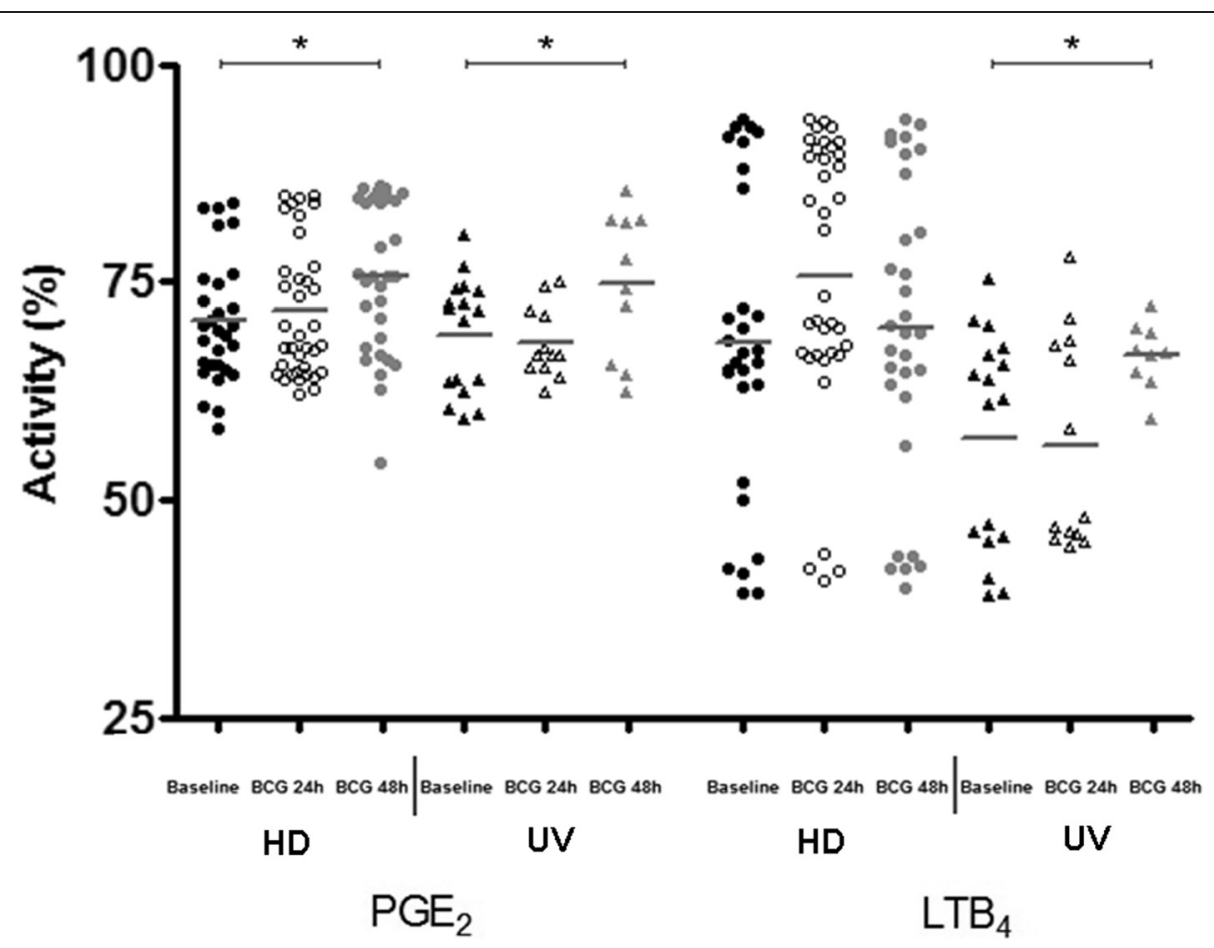

Fig. 2 Prostaglandin E2 $\left(\mathrm{PGE}_{2}\right)$ and leukotriene B4 $\left(\mathrm{LTB}_{4}\right)$ activity levels $(\%)$ in healthy donor $(H D ; n=36)$ and umbilical vein (UV; $\left.n=17\right)$ groups representing baseline and different times of in vitro BCG Moreau infection in human mononuclears. Horizontal bars represent mean values in each condition. ${ }^{*} p \leq 0.05$

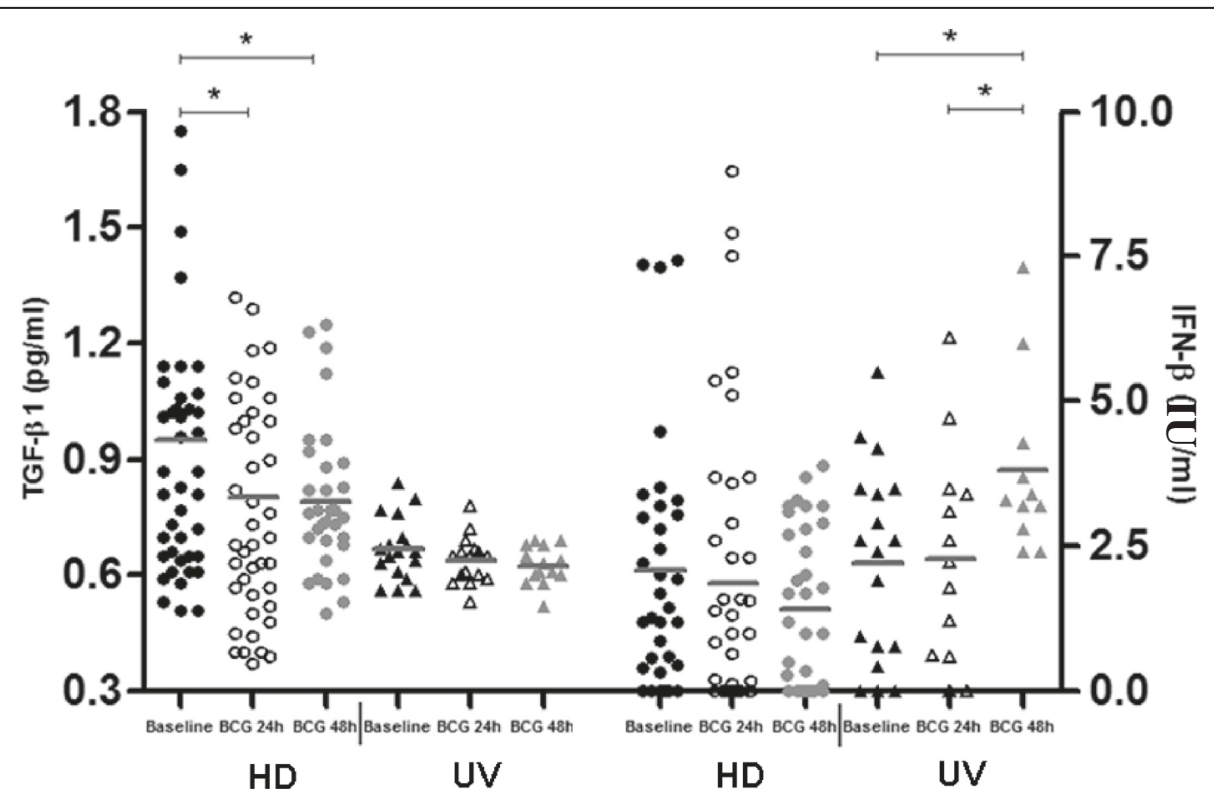

Fig. 3 Transforming growth factor (TGF)- $\beta 1$ levels (pg/ml) and Interferon (IFN)- $\beta$ levels (IU/ml) in healthy donor (HD; $n=42$ ) and umbilical vein $(U V ; n=18)$ groups representing baseline and different times of in vitro BCG Moreau infection in human mononuclears. Horizontal bars represent mean values in each condition. ${ }^{*} p \leq 0.05$ 
Here, consistent data have shown an enhancement of the in vitro apoptosis rate in monocytes from both adult and neonate individuals only after repeated long-term incubation with CM from autologous, infected BCG Moreau cultures that have been regularly used in a previous model. Hence, after a total of $48 \mathrm{~h}$ of infection, BCG stimulated a sufficient output of soluble factors in the $\mathrm{CM}$, in both PBMC and CBMC cultures, to drive additional $120 \mathrm{~h}$ of autologous monocyte cell-death. This result, together with higher in vitro necrosis rate in the UV group, is generally in line with our previous data suggesting the distinct cell-death patterns induced by BCG [8]. That was associated both with higher NO released from the BCG-primed group, but not from the naïve individuals, and also by a distinct pattern of additional mediators and cytokines that found a close parallel to its respective cell-death outcome.

Reactive nitrogen intermediates, such as NO, have been reported to possess anti-mycobacterial activity, and it has long been established [13], as well as currently understood, that $\mathrm{NO}$ is crucial in the host response against intracellular pathogens [14]. Macrophages can directly inhibit the proliferation of mycobacteria by producing NO. In addition to the microbicidal properties, $\mathrm{NO}$ is also important as signaling molecules and therefore promotes macrophage activation and prominent apoptosis. This facilitates antigen presentation and helps to connect the innate to the adaptive immune responses $[13,14]$. Hypothetically, the more apoptosis is induced by a vaccine against $\mathrm{TB}$ in host cells, the more effective is the response against $M$. tuberculosis. However, there is insufficient evidence to determine whether $\mathrm{NO}$ is involved in the defense against the TB bacillus in humans. Here, the higher NO levels released after BCG infection of mononuclears in the HD group, but not in the UV group, might denote indirectly a memory phenotype that remains active for years after priming, thus creating effective, long-lasting protection in those individuals. The balance of cytokines that efficiently upregulate NO production, such as IFN- $\gamma$, TNF- $\alpha$ and IL-1 $\beta$, may play a critical role in the defense mechanism against TB [19].

As above stated, IFN- $\gamma$ synergizes with TNF- $\alpha$ for NO production. Thus, our two previous in vitro studies corroborate with the present one through lower NO levels detected in neonate individuals: Virtually no IFN- $\gamma$ production [20] or TNF- $\alpha$ levels [8] were found in those assessments. Therefore, studying human immune response using the BCG Moreau vaccine and also including the NO production may give us a better understanding of the TB pathogenesis as well.

Resistance or susceptibility to a given infection is a complex phenotype with a multifactorial trait, with several critical candidates playing an important role. As an example, arachidonic acid metabolites converted to the eicosanoids $\mathrm{PGE}_{2}$ and $\mathrm{LTB}_{4}$ are inflammatory mediators which are likely to be involved in apoptosis and necrosis episodes, respectively. Originally, $\mathrm{PGE}_{2}$ was described as the major prostanoid in the lung with an important antifibrotic role. On one hand, $\mathrm{PGE}_{2}$ mediates vasodilatation, increases vascular permeability, enhances pain perception by bradykinin and histamine, alters connective tissue metabolism, and enhances osteoclastic bone resorption. On the other hand, $\mathrm{LTB}_{4}$ causes the accumulation of inflammatory cells in the inflamed sites and degranulation of polymorphonuclear leukocytes [21]. The preferential synthesis of bioactive eicosanoids, mainly prostanoids, is a critical branch point for the innate antimycobacterial response of the infected macrophages. Distinct virulence determinants can manipulate macrophage inflammation toward both extremes, both inducing anti-inflammatory mediator production and also promoting pro-inflammatory TNF [10]. More importantly, $\mathrm{PGE}_{2}$ was recently defined as a pro-apoptotic host lipid mediator which protects against necrosis [9, 10]. In contrast, suppression of $\mathrm{PGE}_{2}$ synthesis by a pronecrotic mediator results in mitochondrial damage and inhibition of plasma membrane repair mechanisms, ultimately leading to the induction of necrosis. Thus, the balance between $\mathrm{PGE}_{2}$ and $\mathrm{LTB}_{4}$, presumably another pronecrotic lipid intermediary, may actually determine whether BCG-infected macrophages undergo apoptosis or necrosis, and this balance determines the outcome of infection. Accordingly, comparable to previous studies, we observed a significant pattern showing that $\mathrm{PGE}_{2}$ and $\mathrm{LTB}_{4}$ were highly correlated to the corresponding cell-death patterns; i.e. BCG-infected mononuclears induced $\mathrm{PGE}_{2}$ and apoptosis in both groups but induced $\mathrm{LTB}_{4}$ and necrosis in the UV group only. Finally, the absence of IL-1 decreases $\mathrm{PGE}_{2}$ in a model of $M$. tuberculosis-infected deficient mice, and the addition-of IL-1 or $\mathrm{PGE}_{2}$ reduces the bacteremia, whereas the TNFinduced $\mathrm{LTB}_{4}$ rises in wild-type animals [18]. Additionally, blocking of type I IFN signaling resulted in increased IL-1 and $\mathrm{PGE}_{2}$. Importantly, protection directly correlated with higher $\mathrm{PGE}_{2}$ and lower type I IFN and IL1Ra production. Likewise, $\mathrm{PGE}_{2}$ also inhibited type I IFN production in $M$. tuberculosis-infected human macrophages. These findings reveal that suppression of type I IFNs and their probacterial activity is a main mechanism of IL-1- and $\mathrm{PGE}_{2^{-}}$ mediated host resistance against $M$. tuberculosis.

Similar to $\mathrm{LTB}_{4}$, IFN- $\beta$ seems to be implicated earlier in necrosis [16] and more recently in necroptosis [17]. In fact, one of the mechanisms underlying enhanced susceptibility to bacterial infections is the stimulation of IFN- $\beta$ production, as stated above, and the latter study showed a Salmonella enterica-induced macrophage death via necroptosis in a type I IFN-dependent manner. That novel cell-death modality is usually triggered by CD95 and 
TNF family of death receptors, and this programmed cell necrosis is distinct from apoptosis [22]. In another setting, $\mathrm{pDCs}$ were shown to be a major cell population producing IFN- $\beta$ through toll-like receptor (TLR) signaling [23]. Generally, pDCs produce most IFN- $\beta$ within $48 \mathrm{~h}$ after pathogen infection and then differentiate into mature DCs with enhanced antigen-presenting capability, after which they become refractory upon secondary stimulation and lose their IFN- $\beta$ production ability. Our current data of highest IFN- $\beta$ levels after that analogous period of BCGinfected mononuclears in the UV group again shows this cytokine to be well correlated with the necroptosis pattern shown by Robinson and colleagues [17]. However, in our system the exact underlying mechanism (necrosis vs. necroptosis) has not yet been fully elucidated.

TGF $\beta 1$ might act as an anti-inflammatory arm prone to damper Th1 immune response. Actually, in line with the current data, our very recent study also failed to find any Th3 cell phenotype induced by the BCG vaccine in the sensitized adult cohort: Comparable TGF $\beta 1$ levels were found regardless of the stimulus employed [24]. That study originally established that the BCG Moreau vaccine induced a significantly elevated IFN- $\gamma /$ IL-10 ratio only in the HD group, confirming a polarized Th1 pattern in vitro.

Based on those previous findings, our current primary results suggest that apoptosis was again induced in monocytes, probably via TNF and/or IL- $1 \beta$ in adults, but not in neonates [8]. Of note, IL- $1 \beta$ drives $\mathrm{PGE}_{2}$ synthesis in infected human macrophages [18]. However, we could not rule out a role for other candidates, such as ATP, TWEAK, TRAMP, TRAIL, and CD95, since there is a long list of potential candidates for apoptosis induction in our system, particularly for the new data regarding naïve individuals [25].

Taking together, one could conclude that the apoptosis pattern found in resting monocytes from both immunized and naïve groups correlates well with high proinflammatory cytokine levels induced in BCG-infected mononuclears. However, necrosis data supports previous findings in neonates only, but additional studies are warranted to rule out other mechanisms such as pyroptosis or necroptosis. These findings support the hypothesis that BCG Moreau strain induces a milieu of soluble factors, which further stimulate distinct cell-death patterns regarding the maturation of the immune system. This pattern may set the stage for a subsequent anti-mycobacterial immune response, which could have profound effects during vaccination.

A possible unifying hypothesis on the central role of cell-death modality in TB pathogenesis is that lesser apoptosis levels in BCG-infected monocytes of neonates could predispose those individuals to the development of inflammatory disease due to lack of protective and specific immune response. This is because only the phagocytosis of apoptotic cells leads to the resolution of inflammation [26]. Conversely, the progress to necrosis could predispose those individuals to the development of disease, since virulent $M$. tuberculosis inhibits apoptosis and triggers necrosis of host macrophages, which delays the initiation of adaptive immunity $[9,10]$. Thus, BCG immunization is the only pathway for increasing the immune response against $M$. tuberculosis. On the other hand, BCGvaccinees show a balance between regulatory and effective immune responses, and a breakdown in this equilibrium would be crucial for TB development.

Despite some tantalizing clues and frequent overstatements, the underlying immunological mechanisms by which the BCG Moreau vaccine evokes protective immune response against $M$. tuberculosis continues to be poorly understood. The central puzzle that remains to be deciphered is: How exactly does the BCG immunization confer partial protection in the vaccinees?

\section{Conclusion}

In this study, BCG-infected macrophages from a naïve group triggered $\mathrm{PGE}_{2}, \mathrm{LTB}_{4}$, and IFN- $\beta$ levels, but BCG triggered copious amount of $\mathrm{NO}$ and $\mathrm{PGE}_{2}$ in adults only. Accordingly, BCG elicited a cell death modality leading to cytolysis in infected macrophages from the naïve group only. In striking contrast, apoptosis was found in the same system in both groups studied. Taken together, our data is in line with other studies demonstrating critical roles for endogenous compounds, such as $\mathrm{NO}, \mathrm{PGE}_{2}, \mathrm{LTB}_{4}$, IFN- $\beta$ and TGF- $\beta 1$, in the regulation and induction of host macrophage cell death patterns and their relevant role in the modulation of inflammation. A better understanding of the factors that protect against TB will help us identify the processes by which this protection is achieved, thus opening up a horizon for its future clinical applicability. Ultimately, because prevention is still one of the best approaches to manage infectious diseases, this leads to the development of improved prophylactic vaccines for $\mathrm{TB}$ that, together with infectious diseases in general, disproportionately affects poor and marginalized populations.

\section{Additional file}

Additional file 1: Figure S1. Connecting lines for (A) Prostaglandin E2 $\left(\mathrm{PGE}_{2}\right)$ and $(\mathrm{B})$ leukotriene $\mathrm{B} 4\left(\mathrm{LTB}_{4}\right)$, in activity levels (\%), and (C) Transforming growth factor (TGF)- $\beta 1$ levels, in $\mathrm{pg} / \mathrm{ml}$, and (D) Interferon (IFN)- $\beta$ levels, in $\mathrm{IU} / \mathrm{ml}$, in healthy donor $(\mathrm{HD} ; n=20)$ and umbilical vein $(U V ; n=6)$ groups representing baseline and different times of in vitro BCG Moreau infection in human mononuclears. (PPT $51 \mathrm{~kb}$ )

\section{Abbreviations}

TB: Tuberculosis; BCG: Bacillus Calmette-Guerin; HD: Healthy donors; UV: Umbilical vein; HIV: Human immunodeficiency virus; CM: Conditioned medium; PBS: Phosphate buffered saline; DMSO: Dimethyl sulfoxide; ELISA: Enzyme linked immunosorbent assay; $\mathrm{PGE}_{2}$ : Prostaglandin E2; $\mathrm{LTB}_{4}$ : Leukotriene B4; IFN: Interferon; TGF: Transforming growth factor; 
NO: Nitric oxide; IL-1: Interleukin-1; IL-10: Interleukin-10; TNF: Tumor necrosis factor; pDC: Plasmacytoid dendritic cell; TLR: Toll-like receptor; PBMC: Peripheral blood mononuclear cell; CBMC: Cord blood mononuclear cell; FACS: Fluorescence-activated cell sorting; ATP: Adenosine triphosphate; TWEAK: TNF-related weak inducer of apoptosis; TRAMP: TNF receptor-related apoptosis-mediating protein; TRAlL: TNF-related apoptosis-inducing ligand; SEM: Standard error of the mean

\section{Competing interests}

The authors declare that they have no competing interests.

\section{Authors' contributions}

$J L, M S$ and TP participated in the design of the study, performed the experiments, and analysed data and statistics. CP, LP and SM carried out several experiments for cell viability and nitric oxide detection, and collected the patient samples. JL and MS provided helpful discussions and edited the manuscript. LRCB provided clinical expertise and helpful discussions. PRZA conceived the study, participated in the design and coordination of the study, analysed data and statistics, wrote the grant application, and wrote the manuscript. All the experiments were performed at the Instituto Oswaldo Cruz (Fiocruz) laboratory. All authors read and approved the final manuscript.

\section{Acknowledgments}

The authors are grateful to Anne F. Carlisle for text editing. We also thank the HUGG staff for their help during the clinical procedures and Vitor C. Lima for his help during technical procedures. This work was partly supported by IOC/FIOCRUZ, and by National Institute of Science and Technology on Tuberculosis (INCT-TB) from CNPq; LP and JS are the recipients of FAPERJ scholarships, TP is the recipient of PEC / FIOCRUZ scholarship, and PRZA is granted with both FAPERJ-JCNE and CNPq-PQ-2 research fellowships.

\section{Received: 19 November 2014 Accepted: 22 October 2015}

Published online: 29 October 2015

\section{References}

1. World Health Organization. Global Tuberculosis Report. 2013.

2. Netto EM, Dye C, Raviglione MC. Progress in global tuberculosis control 1995-1996, with emphasis on 22 high-incidence countries. Global Monitoring and Surveillance Project. 1999;3(4):310-20.

3. Orme IM. The immune response to the cell wall of Mycobacterium bovis BCG. Clin Exp Immunol. 1988;71:388-93.

4. Antas PR, Castello-Branco LR. New vaccines against tuberculosis: lessons learned from BCG immunization in Brazil. Trans R Soc Trop Med Hyg. 2008;102:628-30.

5. Grage-Griebenow E, Durrbaum-Landmann I, Pryjma J, Loppnow H, Flad HD, Ernst M. Apoptosis in monocytes. Eur Cytokine Netw. 1998;9:699-700.

6. Flad HD, Grage-Griebenow E, Petersen F, Scheuerer B, Brandt E, Baran J, et al. The role of cytokines in monocyte apoptosis. Pathobiology. 1999;67:2913.

7. Netea MG, Azam T, Lewis EC, Joosten LA, Wang M, Langenberg D, et al. Mycobacterium tuberculosis induces interleukin-32 production through a caspase-1/IL-18/interferon-gamma-dependent mechanism. PLoS Med. 2006;3:e277.

8. Simas CJ, Silva DP, Ponte CG, Castello-Branco LR, Antas PR. Patterns of in vitro cell-death, metaloproteinase- 9 and pro-inflammatory cytokines in human monocytes induced by the BCG vaccine, Moreau strain. Vaccine. 2011;29:6446-50.

9. Divangahi M, Behar SM, Remold H. Dying to live: how the death modality of the infected macrophage affects immunity to tuberculosis. Adv Exp Med Biol. 2013;783:103-20.

10. Chen M, Divangahi M, Gan H, Shin DS, Hong S, Lee DM, et al. Lipid mediators in innate immunity against tuberculosis: opposing roles of PGE2 and LXA4 in the induction of macrophage death. J Exp Med. 2008:205:2791-801.

11. Nagabhushanam V, Solache A, Ting LM, Escaron CJ, Zhang JY, Ernst JD. Innate inhibition of adaptive immunity: Mycobacterium tuberculosis-induced IL-6 inhibits macrophage responses to IFN-gamma. J Immunol. 2003;171:4750-7.

12. Lima-Junior JC, Rodrigues-da-Silva RN, Pereira VA, Storer FL, Perce-da-Silva DS, Fabrino DL, et al. Cells and mediators of inflammation (C-reactive protein, nitric oxide, platelets and neutrophils) in the acute and convalescent phases of uncomplicated Plasmodium vivax and Plasmodium falciparum infection. Mem Inst Oswaldo Cruz. 2012;107:1035-41.

13. Kwon OJ. The role of nitric oxide in the immune response of tuberculosis. J Korean Med Sci. 1997;12:481-7.

14. Flynn JL, Chan J. Immunology of tuberculosis. Annu Rev Immunol. 2001;19:93-129.

15. Rola-Pleszczynski M, Stankova J. Cytokine gene regulation by PGE(2), LTB(4) and PAF. Mediators Inflamm. 1992;1:5-8.

16. Sheremata WA, Taylor JR, Elgart GW. Severe necrotizing cutaneous lesions complicating treatment with interferon beta-1b. N Engl J Med. 1995;332:1584

17. Robinson N, McComb S, Mulligan R, Dudani R, Krishnan L, Sad S. Type I interferon induces necroptosis in macrophages during infection with Salmonella enterica serovar Typhimurium. Nat Immunol. 2012;13:954-62.

18. Mayer-Barber KD, Andrade BB, Oland SD, Amaral EP, Barber DL, Gonzales J, et al. Host-directed therapy of tuberculosis based on interleukin-1 and type I interferon crosstalk. Nature. 2014;511:99-103.

19. Paziak-Domanska B, Klink M, Jurkiewicz M, Rudnicka W. Production of reactive nitrogen and oxygen intermediates in human granulocytes and monocytes during internalization of live BCG bacilli. Med Dosw Mikrobiol. 2000;52:353-60.

20. Ponte CG, Antas PR, Peres LM, Marinho SP. Comments on the neonatal bacillus Calmette-Guerin vaccination: adding notes in proof of nonspecific effect. Am J Respir Crit Care Med. 2013;187:778-9.

21. Marcinkiewicz J. Regulation of cytokine production by eicosanoids and nitric oxide. Arch Immunol Ther Exp (Warsz). 1997:45:163-7.

22. Liang S, Qin X. Critical role of type I interferon-induced macrophage necroptosis during infection with Salmonella enterica serovar Typhimurium. Cell Mol Immunol. 2013;10:99-100.

23. Cella M, Jarrossay D, Facchetti F, Alebardi O, Nakajima H, Lanzavecchia A, et al. Plasmacytoid monocytes migrate to inflamed lymph nodes and produce large amounts of type I interferon. Nat Med. 1999;5:919-23.

24. Ponte C, Peres L, Marinho S, Lima J, Siqueira M, Pedro T, et al. In vitro T-cell profile induced by BCG Moreau in healthy Brazilian volunteers. Hum Vaccin Immunother. 2015;11:450-7.

25. Elmore S. Apoptosis: a review of programmed cell death. Toxicol Pathol. 2007:35:495-516.

26. Maderna P, Godson C. Phagocytosis of apoptotic cells and the resolution of inflammation. Biochim Biophys Acta. 1639;2003:141-51.

\section{Submit your next manuscript to BioMed Central and take full advantage of:}

- Convenient online submission

- Thorough peer review

- No space constraints or color figure charges

- Immediate publication on acceptance

- Inclusion in PubMed, CAS, Scopus and Google Scholar

- Research which is freely available for redistribution 